\title{
Towards Decisive Garments for Heat Stress Risk Detection
}

\author{
Mitra Baratchi \\ University of Twente \\ Enschede, The Netherlands \\ m.baratchi@utwente.nl

\section{Lennart Teunissen,} \\ Peter Ebben, \\ Wouter Teeuw, \\ Saxion University of Applied \\ Sciences \\ Enschede, The Netherlands \\ $\{$ l.p.j.teunissen, p.w.g.ebben, \\ w.b.teeuw\}@saxion.nl
}

\section{Abstract}

One of the numerous applications of wearable computers is providing safety in occupations where heat-related injuries are prevalent. Core temperature, as a parameter that cannot be measured by on-body

Permission to make digital or hard copies of all or part of this work for personal or classroom use is granted without fee provided that copies are not made or distributed for profit or commercial advantage and that copies bear this notice and the full citation on the first page. Copyrights for components of this work owned by others than ACM must be honored. Abstracting with credit is permitted. To copy otherwise, or republish, to post on servers or to redistribute to lists, requires prior specific permission and/or a fee Request permissions from Permisions@acm

UbiComp/ISWC'16 Adjunct, September 12-16, 2016, Heidelberg,

(C) 2016 ACM. ISBN 978-1-4503-4462-3/16/09..\$15.00 sensors is a variable that is specifically interesting for realizing such applications. In the context of the design of a sensor-shirt that can be used by firefighters, in this paper we study the importance of different types of sensor measurements and their placement for estimating core temperature. We propose a model for inferring the dangerous states of core temperature. Our evaluation results show that our model can to a great extent estimate hazardous situations caused by heat accumulation.

\section{Author Keywords}

Wearable computers; machine learning; ambulatory physiological monitoring; smart-shirt; heat stress; sensor-shirt.

\section{ACM Classification Keywords}

H.5.m. Application-based systems: Miscellaneous.

\section{INTRODUCTION}

Heat stress may cause a person's body temperature to rise above the hyperthermic threshold of $37.8-38.3^{\circ} \mathrm{C}$. Prolonged and/or severe hyperthermia may result in disability or even mortality. This situation is a common casualty among people with occupational heat exposure such as firefighters, mineworkers, and any other type of profession which involves operations in high temperatures, radiant heat sources, high humidity, 


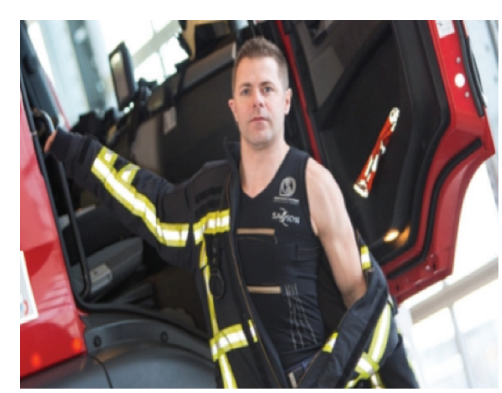

Figure 1: A sensor-shirt for estimating fire-fighters health risks. direct physical contact with hot objects, protective clothing, or strenuous physical activities.

Heat stress is directly detectable by measuring a person's core body temperature. Common ways of measuring core temperature are, however, oftentimes invasive as in swallowing core temperature pills (intestinal), or using rectal probes. Such measurement methods are not practical for use on an occupational basis. Having the possibility of measuring many types of physiological parameters, wearable sensors may provide the potential of estimating core temperature from other measurements. However, it remains an open question which sensors can or should be used for such measurements, and how measurements are to be combined. Difficulties are aggravated when the sensing should take place in an unintrusive fashion.

In the context of design of a sensor-shirt (Figure 1), in this paper, we explore accurate estimation of core body temperature through highly unintrusive wearable sensors. In particular, we concentrate on developing a model that takes an existing dataset containing input from multiple on-body and environmental sensors and ask ourselves: (1) which sensor measurements are important for accurate temperature estimations, and (2) how machine learning techniques can be used to create a model which can capture the hazards of heat exposure. While previous research has focused on accurate estimation of core temperature, our main contribution is showing that although accurate estimation of core temperature may not be easily feasible, a limited number of well-placed on-body sensors can be effectively used to obtain an accurate classification of hazardous and non-hazardous core temperatures.
We organize the rest of this paper as follows. In the following section we will first introduce the related work. In the third section we discuss our research methodology in developing a model. In the fourth section, we subsequently evaluate our proposed solution. We come to conclusions in the fifth section.

\section{RELATED WORK}

The application of wearable technologies targeting the risks of occupational heat exposure has recently gained attention. There are in general two groups of researchers who have focused on dealing with such risks. The first group has focused on designing wearables that can measure physiological parameters that are relevant for heat-related injuries. The focus of such research is in providing a wearable infrastructure that can measure and transmit certain biometrics without further analysis of the data $[5,8,9]$.

The other group of researchers have focused on the important problem of estimating core temperature and physiological strain. For instance, derivative body temperatures have been studied as an estimate for core temperature [10]. In addition, indicators based on internal and external factors (physiological strain and heat stress indices) have been proposed to estimate the thermal load on the body $[3,7]$. All in all, the results of this research suggests that correct estimation of core temperature (especially, in a non-controlled setting) is a challenging problem [11]. When such estimation is needed to be done from wearables, the challenge becomes even bigger. To the best of our knowledge, there is little research proposing solutions for estimating core temperature from non-invasive physiological parameters provided by wearable computers $[2,4]$. The work most in-line with ours is the 


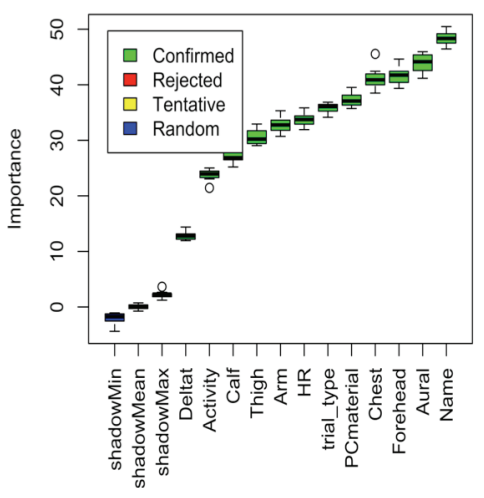

Figure 2: Ranking of different features in prediction of core temperature. (The importance of all features is confirmed and none of them is rejected or considered as tentative) one proposed in [4]. However, the authors have proposed a model for estimating heat-stress risk without considering the importance of each of these sensor measurements. They use the mean skin temperature on various spots as a proxy for core temperature. Nevertheless, none of the previous studies have considered the use of different noninvasive sensors, considering both the operational restrictions, and their placement, as well as, an accurate inference model. These are all essential requirements for designing a sensor-shirt to be used in critical situations.

\section{RESEARCH METHOD}

Our main goal is to predict the occurrence of health risks due to heat stress. As core temperature is not easy to measure directly with sensors, we study the possibility of estimating it through sensors embedded in a shirt. A standard approach for doing so consists of two steps. 1) Feature selection: to study the importance of a set of sensor measurements and their placement in correct estimation of the core temperature. 2) Model development based on the selected measurements: to correctly estimate core temperature. In order to perform the above-mentioned, we use an available multi-parameter dataset provided by [1] which is collected during a fire-fighting training session of 12 male subjects who have performed different activities both in normal $\left(20^{\circ} \mathrm{C}\right)$ and extreme thermal conditions $\left(40^{\circ} \mathrm{C}\right)$. Several parameters are present in the dataset such as heart rate (HR), temperature in different body parts, rectal core temperature, phase change material (PCmaterial), activity type, and the cooling type.

\section{Feature selection: What sensors to choose from?}

By performing feature selection on the dataset, we will decide on the placement of sensors on the shirt such that we have a better estimate of core temperature but also such that the choice of features (which leads to the choice of sensors) is practical with respect to the design of a sensor-shirt. We rank features using a recursive feature selection algorithm in terms of their importance in the estimation of core temperature. Using a feature selection algorithm, many models are trained with different subsets of the features and the best set of features is chosen. For this purpose, we have used an implementation based on Random Forest Algorithm, which adds random features and remodels [6]. The overall ranking of features is presented in Figure 2. As seen in Figure 2, none of the features have been found as unimportant as the importance of all these features is confirmed. Within these features, the most important one in prediction of core temperature are the candidate's identity (Name), and aural temperature. The duration of activity (Deltat) appears to be the least important. Studying these results, and considering the practical consideration of designing a sensor shirt, we choose the selected set of features composed of; trial type, PCmaterial temperature, activity, chest temperature, and HR. The most important features in the estimation of core temperature, which are aural temperature and the candidate's identity, are not considered. The aural temperature cannot be practically measured using a shirt. Considering the candidate's identity implies training the model for every individual who is going to use a sensor shirt. 


\begin{tabular}{c|c|c|c} 
& REG & BNET & NNET \\
\hline ME & 0.003 & 0.0007 & 0.0067 \\
\hline RMSE & 0.4250 & 0.5336 & 0.4101 \\
\hline MAE & 0.2858 & 0.3695 & 0.2757 \\
\hline MPE & 0.0049 & 0.0152 & 0.0177 \\
\hline MAPE & 0.775 & 0.999 & 0.744
\end{tabular}

Table 1: Comparison of accuracy in terms of different error metrics in forecasting core temperature from selected set of features.

\begin{tabular}{c|cc} 
Class & $\begin{array}{c}\text { Selected } \\
\text { Features }\end{array}$ & $\begin{array}{c}\text { Complete } \\
\text { Features }\end{array}$ \\
\hline 35vs.36 & 0.52 & 0.65 \\
\hline $35 v s .37$ & 0.73 & 0.75 \\
\hline $35 v s .38$ & 0.98 & 0.99 \\
\hline $36 v s .37$ & 0.73 & 0.84 \\
\hline $36 v s .38$ & 0.97 & 0.98 \\
\hline $37 v s .38$ & 0.93 & 0.92
\end{tabular}

Table 2: Classification accuracy of different core-temperature classes using complete and selected set of features.

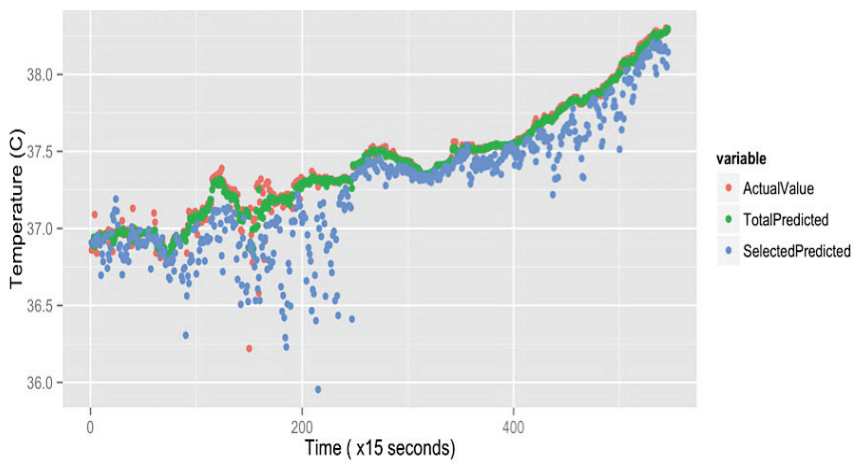

Figure 3: Estimation of core temperature from complete and selected set of features. (TotalPredicted and SelectedPredicted represent prediction from the complete and selected set of features.)

The result of the estimation with a complete and selected set of features using a random forest algorithm is presented in Figure 3. We see that there is a considerable difference between estimations with the complete set of features and the selected set Nevertheless, in the following section we show how this selected set can also be used in creating a model for avoiding heat stress.

\section{Model development: Why estimating core-} temperature is not an option

Having knowledge about core temperature, the heatstress risk can be estimated. Therefore, the most obvious solution in estimating heat stress risk is the correct estimation of core temperature. In this section, we show why such an approach does not work. We formulate a problem to estimate core temperature from a set of other measurable features and we compare different algorithms in solving this problem. The requirement that these algorithms need to satisfy is having the possibility of estimating a numeric output (rather than a categorical one). In other words, they should be regression-based rather than classificationbased. Among many different possible algorithms, we have chosen the following:

Bayesian networks (BNET), which are well-known models for allowing domain expert knowledge as input. To use such input as well, we chose this algorithm. We used the hill-climbing algorithm to learn the model structure and the parameters of the continuous variables are learnt by Gaussian distribution on the discovered model structure. Domain knowledge was further used to refine the model.

Neural networks (NNET), which are less intuitive for domain experts to understand but sometimes lead to better models than Bayesian networks.

Multi-linear regression (REG), which is strong in capturing linear relationships. Pre-analysis of the features showed a considerable linear relationship between variables. Therefore, we also use linear regression to predict the core temperature from the partial set of variables.

We compare the performance of these algorithms in terms of the error metrics mentioned below. In what follows, $a_{t}$ represents the actual value and $f_{t}$ the forecasted value, respectively:

- $\quad$ Root Mean Squared Error (RMSE), provides the sample standard deviation of the difference between the estimated and observed values.

RMSE $=\sqrt{\frac{\sum_{t=1}^{n}\left(f_{t}-a_{t}\right)^{2}}{n}}$ 


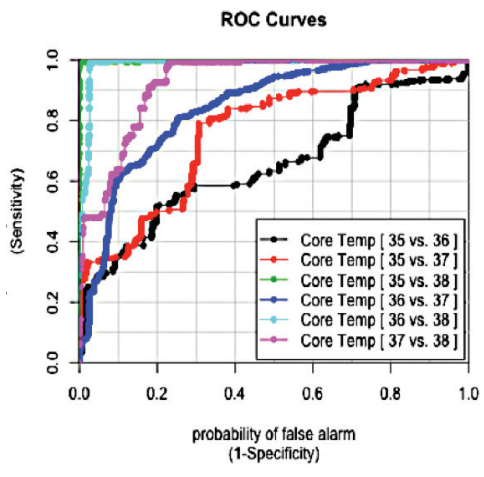

(a)

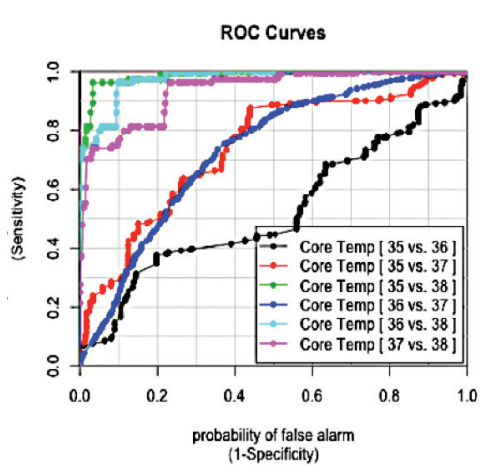

(b)

Figure 4 ROC curves for comparing the classification accuracy of hazardous/safe classes from (a) complete (b) selected set of features. (In the figure "Temp1vsTemp2" refers to classification of temp1 and temp2 from each other)
- Mean Error (ME), also measures the difference between the estimated and actual value in the same unit as the original data. This way models that do consistently under/over estimate are found. $\mathrm{ME}=\frac{1}{n} \sum_{t=1}^{n}\left(f_{t}-a_{t}\right)$

- Mean Absolute Error (MAE), shows how close forecasts are to the eventual outcome. As this metric is measured in the same unit as the data, it is more understandable than the rest of the metrics.

$$
\text { MAE }=\frac{1}{n} \sum_{t=1}^{n}\left|f_{t}-a_{t}\right|
$$

- Mean Absolute Percentage Error (MAPE), is the average of the magnitude of error with respect to the magnitude of the actual value.

$$
\text { MAPE }=\frac{1}{n} \sum_{t=1}^{n}\left|\frac{a_{t}-f_{t}}{a_{t}}\right|
$$

- Mean Percentage Error (MPE), is an average of the percentage errors with respect to the magnitude of the actual value.

$$
\text { MPE }=\frac{100 \%}{n} \sum_{t=1}^{n} \frac{a_{t}-f_{t}}{a_{t}}
$$

Table 1 compares the accuracy of the previously mentioned algorithms in terms of these error metrics. None of the algorithms is consistently better than the rest. As seen, the RMSE of these algorithms which shows the average temperature error, is in range of $0.4^{\circ}$ $\sim 0.5^{\circ} \mathrm{C}$. From the medical point of view, this is a rather large error value and mostly unacceptable for critical medical applications. That is why building a model for accurately estimating the core temperature is not a useful option. The ultimate goal for estimating core temperature is to infer only the hazardous events that will lead to heat stress. Therefore, it is important to only correctly capture the non-hazardous core temperatures from the hazardous ones. Estimation accuracy of lower temperatures does not provide any advantage in an application such as firefighting. A model that can only estimate the temperatures passing the danger threshold and distinguish it from the temperatures below the danger threshold will meet these requirements.

\section{Model development: Heat stress hazard model as an alternative to core temperature estimation}

Our important requirement is that temperatures in the heat stress zone are correctly distinguished from those that are not. Therefore, apart from the previous models that treat core temperature as a numerical variable we perform analysis on core temperature by treating it as a two-class categorical variable; dangerous (above $38^{\circ} \mathrm{C}$ )/safe (below $38^{\circ} \mathrm{C}$ ). In other words, we turn the previous regression problem into a classification problem. To build the new model, we discretized the core temperature to its integer unit range. Next, we built and learnt a Bayesian network on the discretized data.

\section{EVALUATION}

As this is a classification problem, we cannot use the previously mentioned error metrics for evaluation. ROC curves can better be used for evaluation of a classification model through cross-validation. In such curves, a bigger area under the curve would suggest better classification. We have trained the Bayesian network both from the complete and selected set of features resulted from the feature selection phase. ROC curves and the estimation accuracies are presented in Figure 4 and Table 2, respectively. Although using the complete set of features in general might result in a higher area under the curve, the selected set of features can also perform well, especially in 
distinguishing dangerous temperatures from nondangerous ones. In other words, in distinguishing the ones above $38^{\circ} \mathrm{C}$ ( 35 vs 38,36 vs 38,37 vs 38 ).

\section{CONCLUSION}

In this paper, we proposed a model for real-time analysis of physiological parameters for estimation of heat stress risk. Using a dataset collected during a firefighting training session, we studied the importance of different types of sensors, their placement and effectiveness in estimation of core temperature. Our results show that although precise estimation of core temperature with high accuracy is a challenge, it is stil possible to classify core temperature in hazardous and safe zones with high accuracy.

\section{ACKNOWLEDGMENTS}

This research was supported by SaxShirt and MOSES project funded by Tech-For-Future center of expertise HTSM Oost.

\section{References}

1. Brown, F. Physiological, perceptual and cognitive responses to head compared to torso cooling during explosives ordnance disposal activity in moderate and hot conditions. Thesis, Conventry University, 2011.

2. Buller, M. J., Tharion, W. J., and et al. Estimation of human internal temperature from wearable physiological sensors. In
IAAI (2010).

3. Epstein, Y., and Moran, D. S. Thermal comfort and the heat stress indices. Industrial health 44, 3 (2006), 388-398.

4. Gaura, E., Kemp, J., and et al. Leveraging knowledge from physiological data: On-body heat stress risk prediction with sensor networks. Biomedical Circuits and Systems, IEEE Trans. 7, 6 (2013), 861-870

5. Gaura, E. I., Brusey, J., and et al. Increasing safety of bomb disposal missions: a body sensor network approach. Trans. Sys. Man Cyber 39, 6 (2009), 621-636.

6. Kursa, M. B., Rudnicki, W. R., et al. Feature selection with the boruta package, 2010.

7. Niedermann, R., Wyss, E., and et al. Prediction of human core body temperature using non-invasive measurement methods. Int. J. of biometeorology 58, 1 (2014), 7-15.

8. Salim, F., Prohasky, D., and et al. Design and evaluation of smart wearable undergarment for monitoring physiological extremes in firefighting. In Proc. of the 2014 ACM ISWC:Adj, (2014), 249-254.

9. Talavera, G., and et al. Protecting Firefighters with Wearable Devices, vol. 7656 of LNCS. Springer Berlin Heidelberg, 2012, book section 65, 470-477.

10. Teunissen, L., Klewer, J., and et. al. Non-invasive continuous core temperature measurement by zero hea flux. Physiological measurement 32, 5 (2011), 559.

11. Xu, X., Karis, A. J., and et al. Relationship between core temperature, skin temperature, and heat flux during exercise in hear, European Journal of Applied Physiology 113, 9 (2013), 2381-2389. 